\title{
Review of the Current Trends in Clinical Trials Involving Induced Pluripotent Stem Cells
}

\author{
Jennifer Yejean $\mathrm{Kim}^{1,2} \cdot$ Yoojun $\mathrm{Nam}^{2} \cdot$ Yeri Alice $\mathrm{Rim}^{2} \cdot \mathrm{Ji} \mathrm{Hyeon} \mathrm{Ju}^{2,3}$ (이 \\ Accepted: 9 September 2021 / Published online: 16 September 2021 \\ (C) The Author(s), under exclusive licence to Springer Science+Business Media, LLC, part of Springer Nature 2021
}

\begin{abstract}
In 2006, the induced pluripotent stem cell (iPSC) was presented to the world, paving the way for the development of a magnitude of novel therapeutic alternatives, addressing a diverse range of diseases. However, despite the immense cell therapy potential, relatively few clinical trials evaluating iPSC-technology have actually translated into interventional, clinically applied treatment regimens. Herein, our aim was to determine trends in globally conducted clinical trials involving iPSCs. Data were derived both from well-known registries recording clinical trials from across the globe, and databases from individual countries. Comparisons were firstly drawn between observational and interventional studies before the latter was further analyzed in terms of therapeutic and nontherapeutic trials. Our main observations included global distribution, purpose, target size, and types of disorder relevant to evaluated trials. In terms of nontherapeutic trials, the USA conducted the majority, a large average number of participants-187-was included in the trials, and studies on circulatory system disorders comprised a slightly higher proportion of total studies. Conversely, Japan was the frontrunner in terms of conducting therapeutic trials, and the average number of participants was much lower, at roughly 29. Disorders of the circulatory, as well as nervous and visual systems, were all studied in equal measure. This review highlights the impact that iPSC-based cell therapies can have, should development thereof gain more traction. We lastly considered a few companies that are actively utilizing iPSCs in the development of therapies for various diseases, for whom the global trends in clinical trials could become increasingly important.
\end{abstract}

Keywords Clinical trials $\cdot$ Induced pluripotent stem cells $\cdot$ Cell therapy $\cdot$ Interventional $\cdot$ Therapeutic $\cdot$ Nontherapeutic $\cdot$ Allogeneic · Autologous

\section{Introduction}

Stem cells are pluripotent, self-renewable cells with the capability of differentiating into various somatic cells [1-4]. The notion of stem cell research was first introduced in the

Jennifer Yejean Kim and Yoojun Nam authors contributed equally to this work.

Ji Hyeon Ju

juji@catholic.ac.kr

1 Department of Biology, Georgetown University, Washington, DC, USA

2 CiSTEM Laboratory, Catholic iPSC Research Center, College of Medicine, The Catholic University of Korea, Seoul, Korea

3 Division of Rheumatology, Department of Internal Medicine, St. Mary's Hospital, Institute of Medical Science, College of Medicine, The Catholic University of Korea, Seoul, Korea 1960s, from experiments conducted on mouse bone marrow [5]. Continued research in subsequent years culminated in the first isolation of human embryonic stem cells (hESCs), by James Thomson in 1998 [6]. Thereafter, in 2006, Shinya Yamanaka managed to successfully reprogram adult somatic cells into pluripotent stem cells using four reprogramming factors - referred to as Yamanaka factors - namely, octamerbinding transcription factor 4 (Oct4), Krüppel-like factor 4 (KLF4), sex-determining region Y-box 2 (SOX2), and c-Myc [7, 8]. The stem cells derived by Yamanaka, now known as induced pluripotent stem cells (iPSCs), have been studied ever since to increase our understanding of the pathogenesis of various diseases, and aid in developing novel treatment methods [9-11].

$\mathrm{hESCs}$ have been used in several studies and thereby have shown success in clinical trials initially [12-14], though their usage has been challenged. First, since the hESCs are associated with using human embryos at the blastocyst stage, 
they are associated with ethical considerations [15]. Additionally, since ESCs are initially isolated from blastocysts that were not implanted, they are never of autologous origin, hence may elicit immunological rejection if injected into the patients. Hence, the introduction of iPSCs has presented great potential, since unlike hESCs, iPSCs present no ethical concerns $[15,16]$. Since being introduced in 2006, iPSCs have been heavily utilized in hopes of finding novel therapeutic approaches to, and studying the pathogeneses of, a host of disease conditions [17-22]. To this end, innumerable publications are available that cover both in vitro [23-26], and in vivo [27-29] pre-clinical studies using iPSCs.

There have been considerable efforts to organize trends in stem cell research on a clinical-trial basis [30-33], though these studies have either not focused on iPSCs, or relied on only one database from which to draw conclusions. In this article, we have analyzed results from multiple clinical trial registry databases and focused specifically on the utilization of iPSCs as a first-line inclusion criterion. We have also schematically explained the basics of the iPSC-based clinical trial evaluation method we had followed and provided some detailed examples of relevant clinical trials, to enhance comprehension. Moreover, we have provided information on a few companies that utilize iPSCs in developing therapeutic approaches to various diseases, providing further insight into the real-life application potential of such studies.

\section{Literature and Database Search}

The main database sources of multi-trial research data are ClinicalTrials.gov (https://clinicaltrials.gov/) and the International Clinical Trials Registry Platform (ICTRP) (https:// www.who.int/clinical-trials-registry-platform), which is managed by the World Health Organization. To portray a more accurate representation of current global clinical trialrelated trends, our study evaluated the clinical registry platforms of all countries recorded in the International Committee of Medical Journal Editors (ICMJE). We identified 112 suitable trials from ClinicalTrials.gov, by searching for keywords "iPSC" and "induced pluripotent stem cells." From ICTRP, we initially identified 31 trials, though 13 projects were repeated in the results and therefore eliminated, leaving 18 trials for further analysis. Searching for the same keywords against individual country-specific registries. We have also included primary clinical trials registries of countries that are a part of the International Committee of Medical Journal Editors (ICMJE) that do not overlap with clinicaltrials.gov and ICTRP. Such search yielded 7 additional suitable trials, making up a total of 137 clinical trials, which entered into the first pool of analysis. Hence, we have attempted to recapitulate government-based clinical trial registries known to the public. The search was finalized on January $11^{\text {th }}, 2021$.
To ensure accurate representation of the current trends in clinical trials using iPSCs worldwide, three filtering criteria were set. First, projects that did not use iPSCs or made the use thereof optional-were excluded due to the uncertainty of whether iPSCs had, in fact been employed-were eliminated. Based on the initial filtering criteria, 21 projects from ClinicalTrials.gov were eliminated, of which 11 proposed optional use of iPSCs, and the remaining 10 did not include iPSCs in the studies at all. Therefore, 116 studies-including all stages of trial status - with at least minimal involvement of iPSCs, entered the second pool of analysis.

From the second pool, projects that involved iPSCs but did not emphasize them as the primary outcome of the study, were omitted, leaving only projects in which iPSCs were the main focus of the study, for placement in the next pool of analysis. The second round of filtering resulted in the removal of 13 projects originally obtained from ClinicalTrials.gov, leaving 103 studies primarily focused on iPSCs, across all recruitment status stages, for the next evaluation step.

Finally, projects that were ongoing at the time of our evaluation were selected for deeper analysis. Accordingly, projects that had been suspended, terminated, withdrawn, or were of unknown status-defined according to the guidelines provided by ClinicalTrials.gov (https://prsinfo.clini caltrials.gov/definitions.html)—were excluded. A further 19 studies originally obtained from ClinicalTrials. gov were filtered out, of which two had been suspended, seven terminated, one withdrawn, and nine were of unknown status. Similarly, three suspended projects listed on ICTRP were disregarded, leaving 81 observational and interventional clinical trials as relevant on January 11, 2021, for further analysis. The overview of literature and database search schematics is provided in Fig. 1.

\section{Current Therapeutic Clinical Trials using iPSCs}

As seen in Fig. 1, we selected 81 observational and interventional clinical trials from the initial pool of 137 relevant iPSC-related trials that offered keyword search matches, for further analysis (Fig. 2A). For a detailed list of all clinical trials, please refer to Supplementary Data 1.

In our analyses, we sought to understand the trends in clinical trials involving therapeutic usages of iPSCs. In this regard, some of the clinical trials deemed as "interventional" by the authors actually entailed procedures involving extractions of cells from patients, such as skin biopsy and blood withdrawals. Since these projects did not meet our criteria for an interventional clinical trial-trials that implement iPSCs in interventional procedures-we decided to re-organize the studies into two categories: nontherapeutic 
Fig. 1 Classification of global clinical trials using iPSCs in their studies

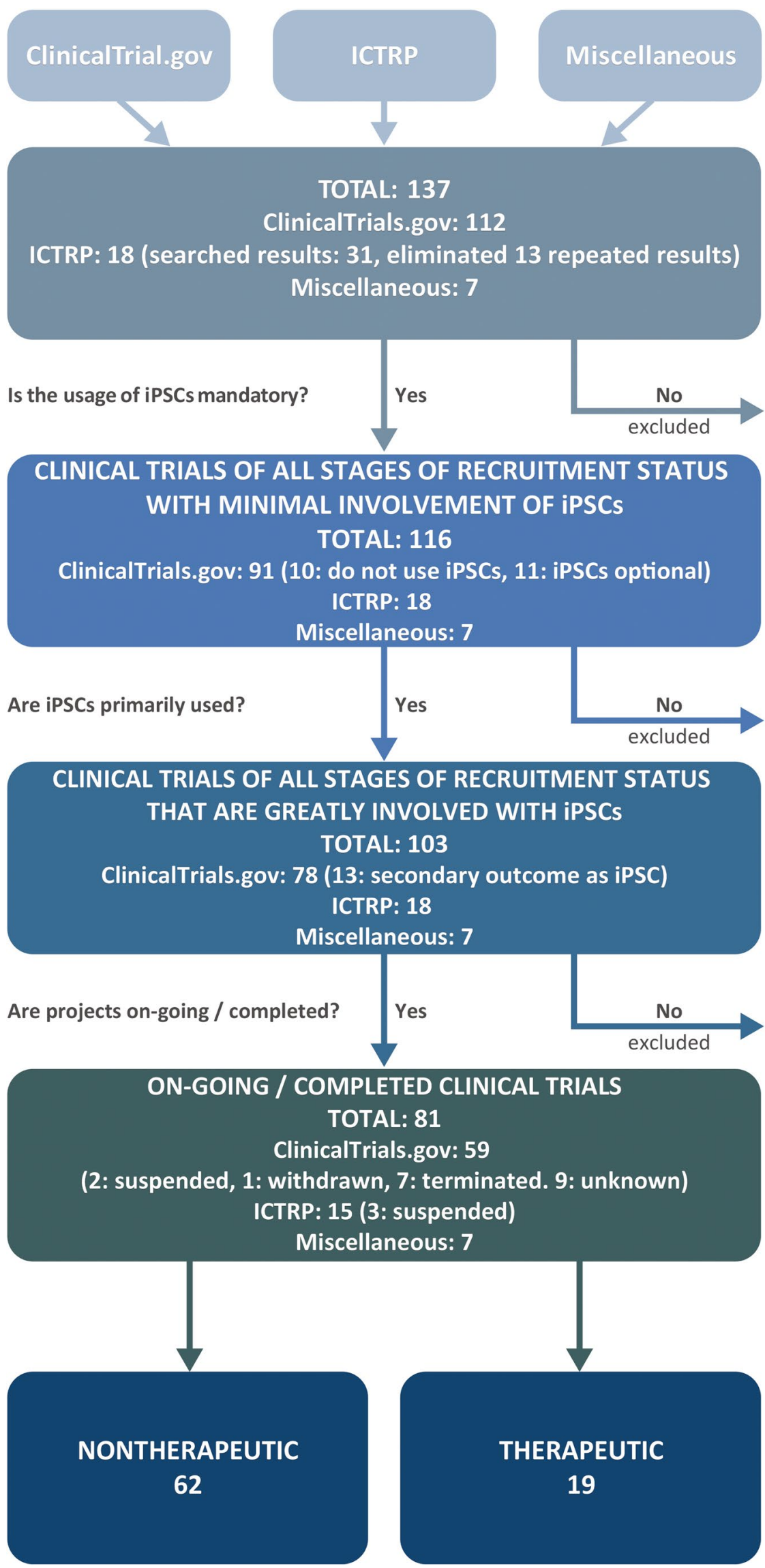


Fig. 2 Distribution of clinical trials involving iPSCs accordingly to different categories. A Initial classification of 81 clinical trials into observational and interventional studies as addressed by the authors. B New classification of 81 clinical trials into nontherapeutic and therapeutic studies as defined earlier. C Classification of nontherapeutic clinical trials according to their use. D Worldwide distribution of nontherapeutic studies. E Classification of studies according to the category of targeted disease
A

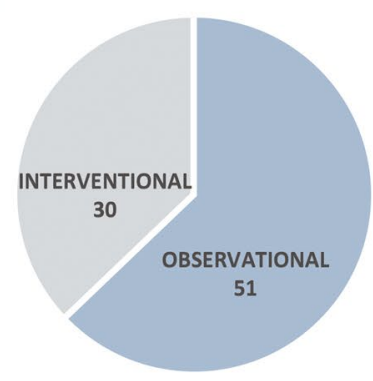

B

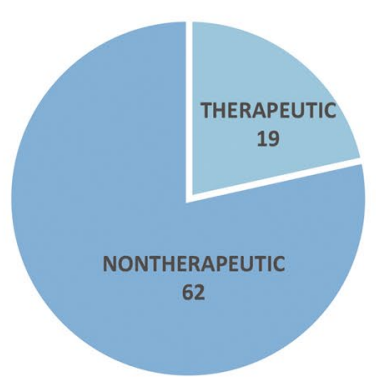

C

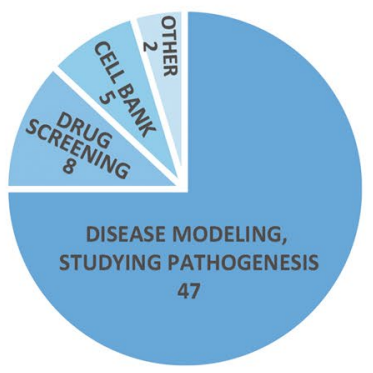

D

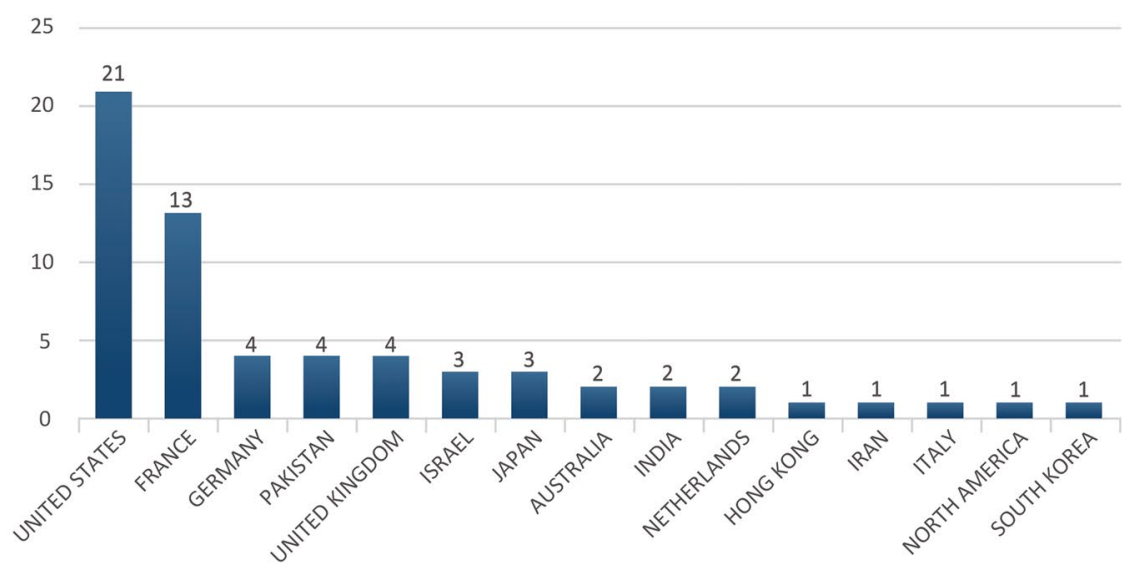

E

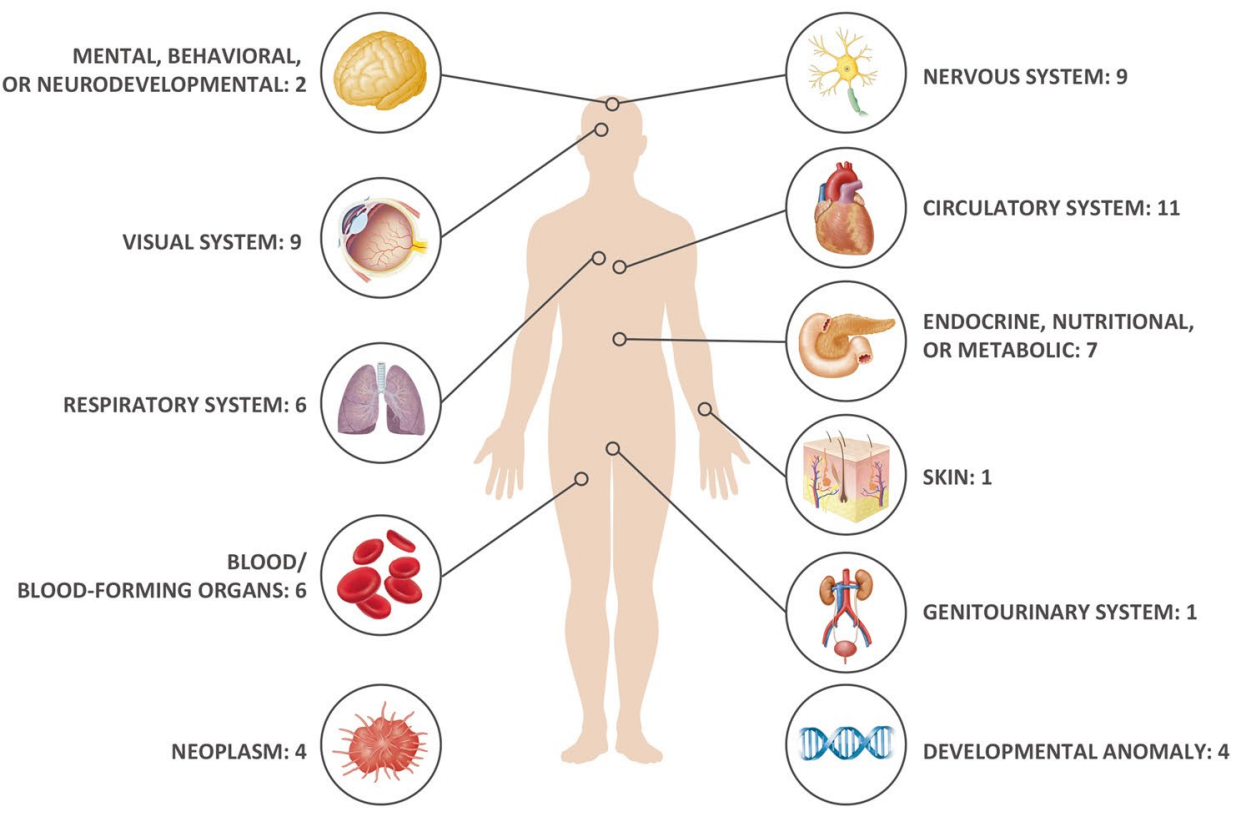

OTHER: 1

and therapeutic studies. We have defined "nontherapeutic" studies as clinical trials that primarily utilize iPSCs in their studies, yet they are not used as treatments but rather used as a method of studying the diseases, such as disease modeling, drug screening, and creating cell banks (Fig. 2C). On the other hand, we have defined "therapeutic" studies as clinical trials in which iPSCs were (re)transplanted into patients in an effort to develop treatments. Ultimately, we have identified 62 nontherapeutic studies and 19 therapeutic studies (Fig. 2B). 


\section{Nontherapeutic Studies}

From 81 "observational" and "interventional" clinical trials, $62(76.5 \%)$ projects were classified as being nontherapeutic, whereas 19 (23.5\%) were therapeutic in nature (Fig. 2B). These clinical trials were further analyzed in terms of global distribution, purpose, target size, and type of disorder, which were classified according to the International Classification of Diseases, $11^{\text {th }}$ revision (ICD-11) guidelines provided by the World Health Organization (https://icd.who.int/brows e11/l-m/en).

The application of iPSCs was variable across studies. Forty-seven studies (75.8\%) utilized iPSCs for disease modeling by differentiating gathered iPSCs into specific cells and studying the disease pathogenesis to ultimately develop treatment options. Eight studies focused on drug screening (12.9\%), and five $(8.1 \%)$ on developing cell banks for further use in future research. Two studies were designated as "other," of which one (1.6\%) used iPSCs in clinical application and the other (1.6\%), on testing the eligibility of various assessments (Fig. 2C).

In terms of the global distribution of ongoing nontherapeutic studies, the United States contributed the highest number-21 (33.3\%)—followed by 13 trials in France (20.6\%). Germany, Pakistan, and the United Kingdom contributed four $(6.3 \%)$, respectively, whereas Israel and Japan each had three (4.8\%). Australia, India, and the Netherlands each presented two (3.2\%), whilst the remaining countries/ continents-Hong Kong, Iran, Italy, North America, and South Korea-had one (1.6\%) each (Fig. 2D). The overall average number of participants in the studies we evaluated, was 187 , whilst the average number of participants per country with more than one ongoing study, was as follows: 315 in the United States, 155 in the Netherlands, 105 in France, 80 in Pakistan, 61 in Israel, 57 in India, 37 in Germany, 24 in Japan, and 18 the United Kingdom.

The nontherapeutic studies included in our evaluation targeted a range of diseases, classified per ICD-11 categorization. We found that disorders of the circulatory system were targeted the most, comprising 11 studies (17.7\%). Thereafter followed disorders of the nervous and visual systems, such as Parkinson's disease and retinal degradation, with nine studies $(14.5 \%)$. Seven studies (11.3\%) focused on endocrine, nutritional, or metabolic disorders, whereas six (9.7\%) each were allocated to disorders of the respiratory system, as well as blood disorders or developmental anomalies of blood-forming organs. Neoplasms and congenital abnormalities were studied in four cases each (6.5\%), and two studies (3.2\%) focused on mental, behavioral, or neurodevelopmental disorders. One trial (1.6\%) studied skin disorders, and another single trial was focused on disorders of the genitourinary system. One study was classified under the category "other" as it was aimed at gathering healthy iPSCs, rather than targeting a certain disorder (Fig. 2E).

\section{Therapeutic Studies}

Of the 81 clinical trials that met the criteria for inclusion in our research, 19 studies (23.4\%) are identified as "therapeutic" (Figs. 2B and 3A). Of the 19 therapeutic clinical trials evaluated, allogeneic iPSCs were mostly used -15 studies (79\%)—whilst autologous iPSCs, derived from patients and injected back to the donor, were used in the remaining four trials (Fig. 3B). 7 studies (37\%) out of 19 therapeutic studies were in Phase I and five were classified as Phase I/II clinical trials, according to the authors. Only one study (5\%) was in Phase III, and in two more (11\%), the phase was not specified. The authors provided no phase-related information on the remaining four studies (21\%) (Fig. 3C).

The trends in therapeutic studies were clearly distinguishable from those in nontherapeutic studies. Compared to that of nontherapeutic clinical trials, the overall average number of participants in an interventional clinical trial was much lower, at 28.7. Divided per country, the averages were as follows: 95.0 in the United States, 48.0 in China, 32.0 in Iran, 14.5 in Australia, 6.0 in Germany, and 5.1 in Japan (Fig. 3D).

Furthermore, Japan was the overwhelming leader in terms of the global distribution of trials, executing more than half-10 studies - of the relevant studies worldwide. This clearly indicates the apparently preferred trial focus area for this country, since a mere $4.8 \%$ of the total nontherapeutic studies were conducted in Japan, as opposed to $52.6 \%$ of relevant therapeutic studies. Conversely, the United States, which presented with the highest number of recurring nontherapeutic studies, was in second place in terms of therapeutic trials, but only conducted four studies (21.1\%), which is significantly lower than their share of nontherapeutic studies. Two studies (10.5\%) were contributed by Australia, whilst China, Germany, and Iran presented one each (5.3\%). Many countries that had conducted several observational studies, including the United Kingdom, France, and Pakistan, offered no registered interventional studies (Fig. 3E).

Similar to the trends seen in observational studies, disorders of the circulatory, as well as nervous and visual systems, were studied the most, comprising four studies (21.1\%) each. Three projects (15.8\%) focused on neoplasms and two $(10.5 \%)$ studied the respiratory system, with specific reference to COVID-19. Disorders of the immune system, as well as blood or blood-forming organs, were dealt with in one study (5.3\%), respectively (Fig. 3E). Further explanations on therapeutic, interventional studies are provided in Table 1. 
Fig. 3 Distribution of therapeutic clinical trials involving iPSCs, according to different categories. A Categorization of interventional clinical trials depending on application of iPSCs. B Distribution according to the type of iPSCs used. C Distribution per clinical trial phase. D Global, averaged distribution of the number of participants. E Worldwide distribution of therapeutic studies. F Classification of studies according to the category of targeted disease
A

B
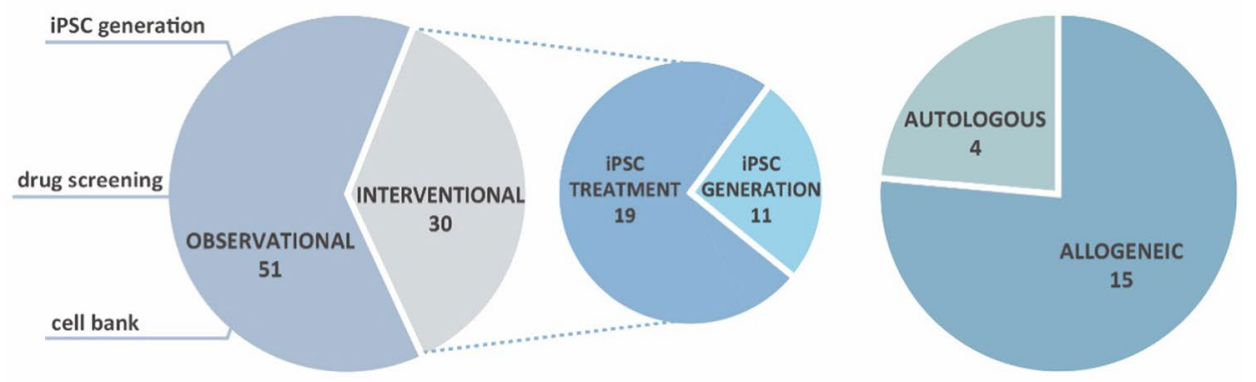

C

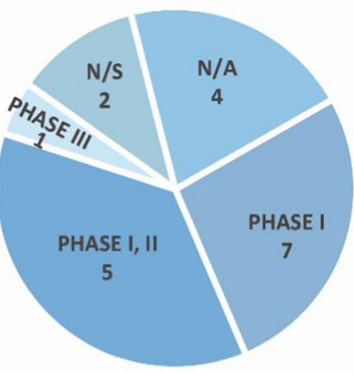

D

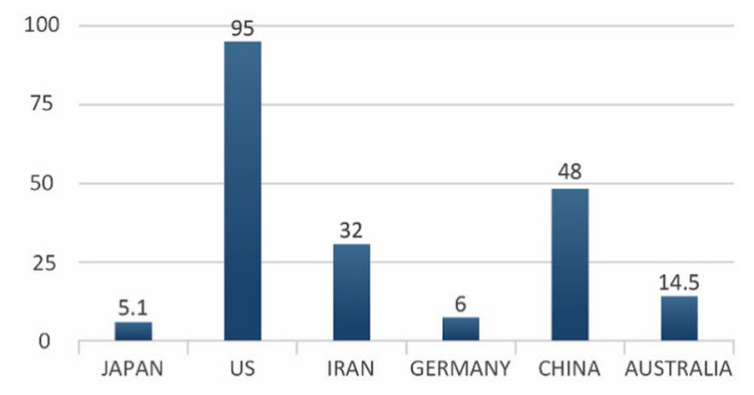

E

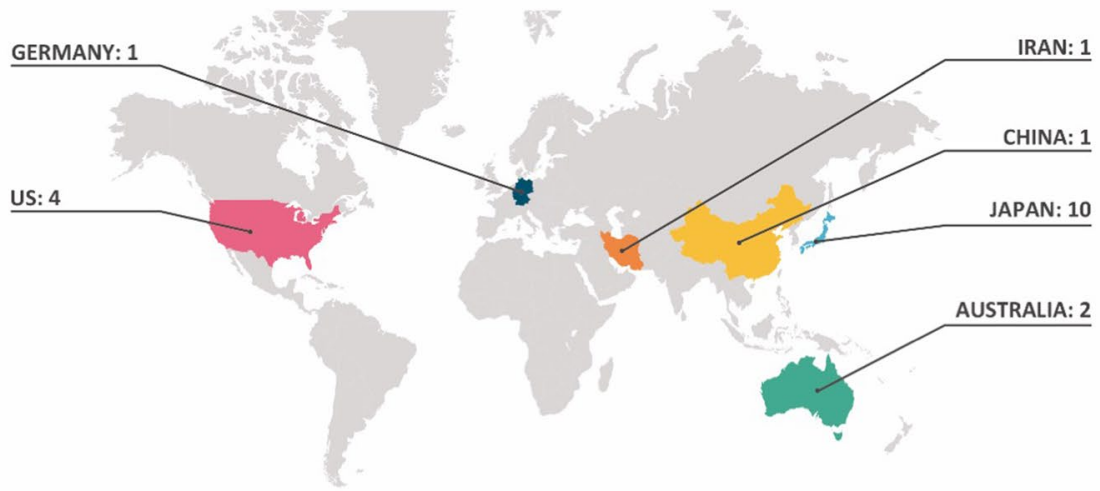

$\mathbf{F}$

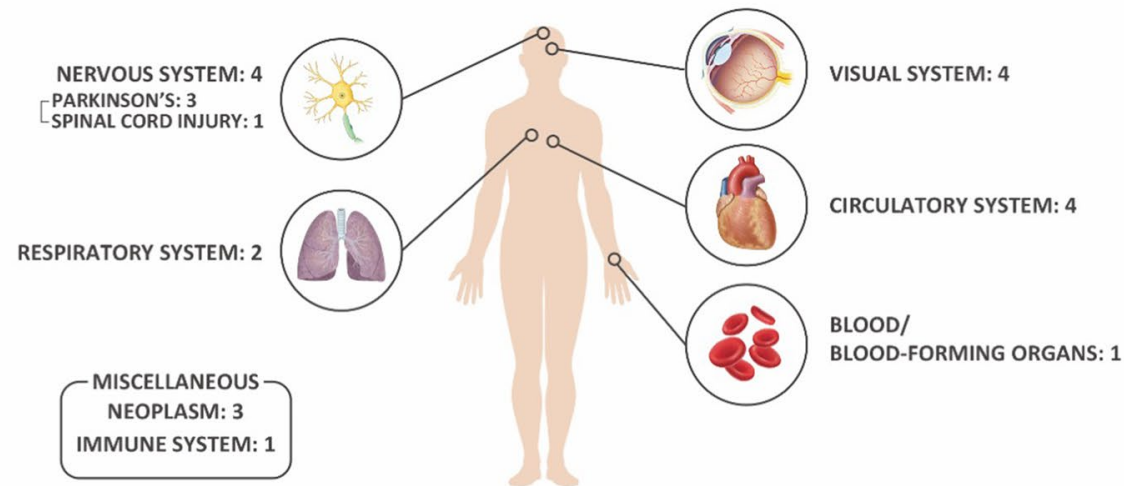




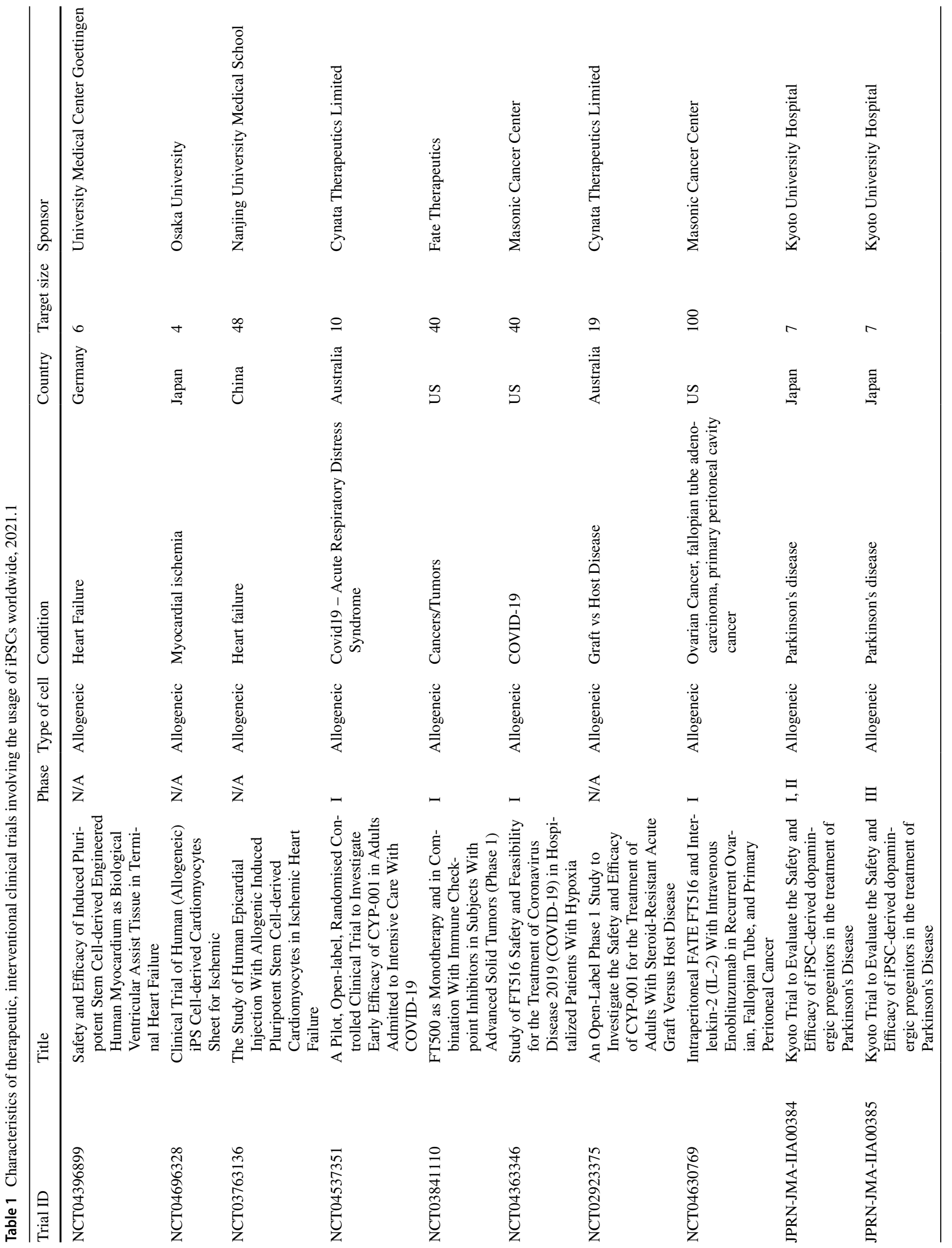




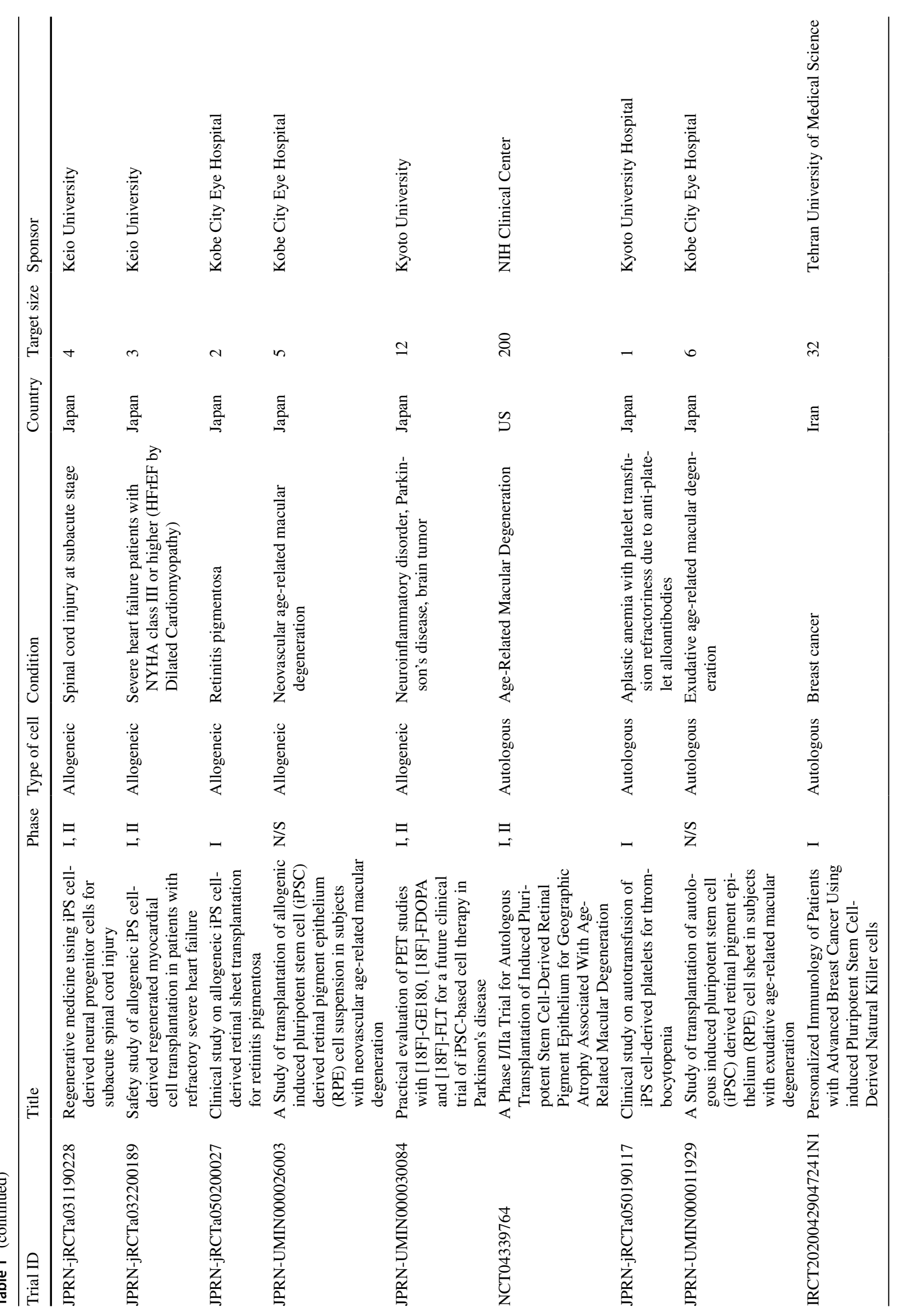




\section{Future Perspectives on Therapeutic Clinical Trials using iPSCs}

In the therapeutic market, iPSCs are giving rise to a wide range of therapeutic possibilities, considering that treatmentspecific target cells differentiated from iPSCs can be directly transplanted into patients. Additionally, biomaterials can be combined with the cells using tissue engineering technology, to enhance the viability of the differentiated cells [34]. Moreover, iPSCs can be utilized to correct mutations when developing clinical treatments for specific genetic diseases [35], and the use of autologous iPSCs negates the risks of immune rejection and related complications [36, 37]

However, the reprogramming of somatic cells into iPSCs requires extensive time and effort. Therefore homozygous iPSCs are preferred over their autologous counterparts, in the development of treatment regimens and the current, ongoing global focus is on generating homozygous iPSC lines. Further to this, many resources are dedicated to the development of human leukocyte antigen (HLA)-related CRISPR technology [36, 37], to reduce immune rejection, and tumorigenicity is a concerning factor in using iPSCs in disease treatment [38-40]. It is thus clear that there are numerous barriers to entry in the field of iPSC-based clinical treatment. Details of current iPSC-based clinical studies are depicted in Table 1. Among those listed, four projects using iPSCs presented completed clinical trials at the date of data extraction, as follows:

1. Clinical trial sponsored by Cynata Therapeutics Limited in Australia, involving the use of CYP-001-an iPSC-derived mesenchymal stem cell (MSC) - to target "Graft-versus-host disease" (GVHD). In 2018, the phase 1 clinical trial was successfully completed in 16 patients with steroid-resistant GVHD, and the results were positive [41].

2. Clinical trial sponsored by Kobe City Eye Hospital in Japan, involving transplantation of allogeneic iPSCderived retinal pigment epithelium (RPE) cells, to treat patients with neovascular age-related macular degeneration (AMD). Scientists transplanted a sheet of iPSCderived corneal cells into the patient's cornea, and when they checked it out a month later, it was confirmed that the patient's eyesight improved [7, 42, 43].

3. Clinical trial sponsored by Kyoto University in Japan, using iPSC-derived dopaminergic neurons to target Parkinson's disease. They will follow their progress for two years and are currently enrolling patients with a target of seven total [44].

4. Clinical trial sponsored by Kobe City Eye Hospital in Japan, in which autologous iPSC-derived RPE cells were transplanted into patients with exudative AMD. A 77-year-old woman with AMD was transplanted with a sheet of iPSC-RPE cells. One year after, the degeneration process stopped, the photoreceptor recovery site was observed, and the patient's vision remained stable [45].

Despite iPSCs proposing many possibilities, very few iPSC-based therapeutic agents are currently being used in clinical practice. However, since iPSCs can theoretically be differentiated into any cell type, scientists worldwide continue to research options to develop treatments using iPSCs. It is therefore expected that the development of iPSC-based treatments will gain momentum, regardless of the process being slow at present.

As with any cell therapy-related clinical trial, development of iPSC-therapeutics is necessarily preceded by tests verifying the level of tumorigenicity, dose toxicity, distribution, and immunogenicity. Additionally, the treatment efficacy must first be confirmed in diseased animal models; unlike that of MSC treatments, the safety of iPSC treatments has not yet been explicitly verified, necessitating more precise evaluation thereof, especially in the case of tumorigenicity tests.

Dr. Masayo Takahashi of Riken Center in Japan, is the pioneer in the field of clinical trials using iPSCs to treat retinal degenerative diseases. She led the first team to successfully transplant autologous iPSC-derived RPE cell sheets into a patient with AMD, in 2014 [7, 42, 43]. This transplant achieved a therapeutic effect without immune rejection or tumorigenic symptoms. Their continued research culminated in the successful transplantation of allogeneic iPSC-derived RPE cells, in March 2017, and they are presently conducting studies on the transplantation of corneal cells derived from iPSCs.

Globally, diseases that have been targeted for iPSCrelated treatment, or on which iPSC-based clinical trials have been completed, are as follows:

\section{Ischemic Cardiomyopathy}

Following in Dr. Takahashi's footsteps, numerous further clinical trials involving iPSCs have been conducted in Japan. For instance, Kawamura et al. conducted transplantation of iPSC-derived cardiomyocytes into a porcine ischemic cardiomyopathy model, achieving improved heart function [44, 46, 47]. Moreover, Osaka University received approval for conducting a clinical trial in which allogeneic iPSC-derived tissue sheets will be transplanted into a human heart [48]. Likewise, Kyoto University received approval for a blood transfusion clinical trial using platelets extracted from iPSCs. 


\section{Parkinson's Disease}

In Japan, dopamine precursors were obtained from iPSCs and their efficacy was confirmed in a rat model [49]. In October 2018, clinical trials began that used these validated iPSC-derived cells for implantation into the brains of patients with Parkinson's disease, who were in their fifties [49].

\section{Natural Killer Cells}

Historically, various strategies have been implemented in attempts to conquer cancer. In 2016, iPSCs-derived natural killer (NK) cells were demonstrated to be effective against ovarian cancer in an animal model [50]. Researchers at the University of Minnesota, and Fate Therapeutics in the United States, have secured US-FDA approval to conduct clinical trials using iPSC-derived NK cells and are working to verify its efficacy against various cancers [51, 52].

\section{GVHD}

Current developments in the treatment of GVHD concur that the use of iPSCs holds much potential. The Australian stem cell and regenerative medicine company, Cynata Therapeutics, has been granted approval to create MSCs using iPSCs and conduct clinical trials on their use. Based on the positive results from Phase I clinical trials, there is anticipation that iPSC-derived MSCs would result in GVHD treatments that are more effective than MSC-based treatments used in the past.

\section{Spinal Cord Injury}

At Keio University in Japan, the effects of using neural progenitor cells derived from iPSCs were evaluated. In February 2018, a clinical trial was approved, using these cells in the treatment of patients with spinal cord injury.

\section{Other Disorders}

The University Medical Center Göttingen in Germany engineered iPSC-derived myocardium to target terminal heart failure, whilst the medical school of Nanjing University in China also worked on developing therapeutic agents against heart failure, using cardiomyocytes derived from iPSCs. Cynata Therapeutics and the University of Minnesota, sponsored by the Masonic Cancer Center (United States) have both started developing cell therapies for COVID-19 treatment.

\section{Commercialization of Prospective Therapeutic Clinical Trials using iPSCs}

There are more than 200 companies worldwide that directly or indirectly use iPSCs, but only a few aims to develop iPSCbased cell therapy products, as seen in Fig. 4. These include:

- BlueRock Therapeutics, recently acquired by Bayer AG, which focuses on developing treatments for Parkinson's disease and heart failure.

- Semma Therapeutics, which was founded with the aim of developing options to treat patients with Type 1 diabetes, using iPSCs.

- Cynata Therapeutics, which focuses on developing treatments against GVHD, using allogeneic iPSCs. Moreover, they are conducting a Phase III clinical trial using CYP004 to treat patients with osteoarthritis and are preparing to run a COVID-19 clinical trial as well.

- The Japanese company, Healios K.K., is preparing a clinical trial to assess treatment options for patients with AMD, in collaboration with Sumitomo Dainippon Pharma.

- Fate Therapeutics with their focus on using clonal master engineered iPSC lines to develop CAR T-cell therapy

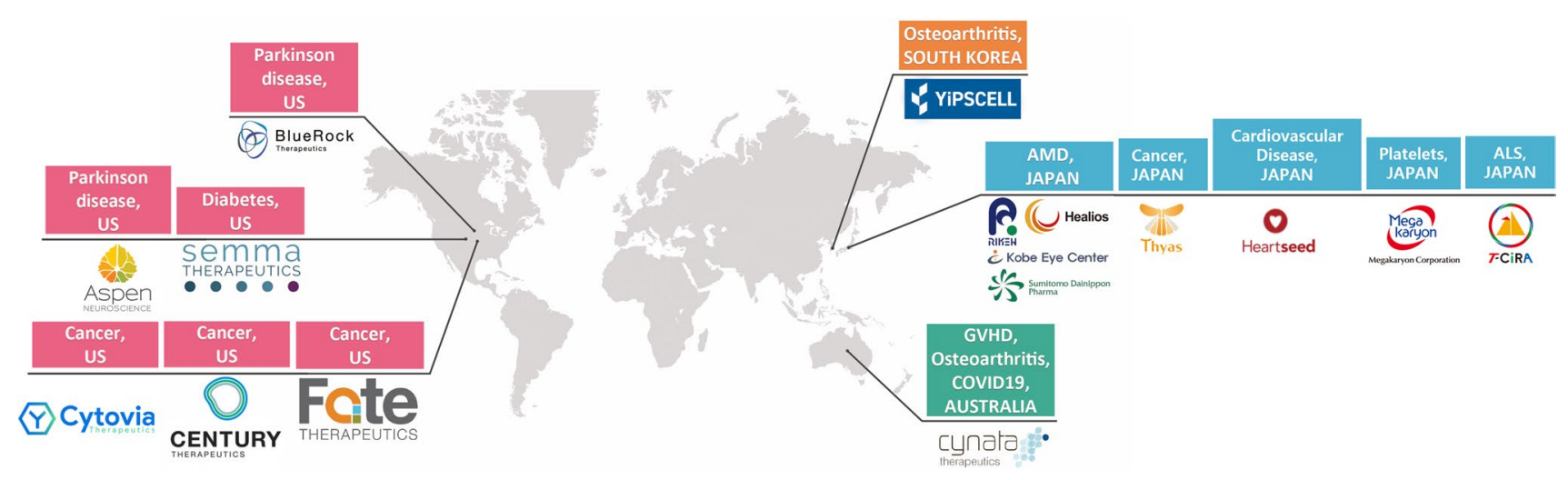

Fig. 4 Companies that aim to develop cell therapy products using iPSCs 
options for the treatment of hematological malignancies and tumors.

- YiPSCELL in Korea that have embarked on developing an iPSC-derived osteoarthritis treatment, marketed as MIUChon ${ }^{\mathrm{TM}}$.

- Aspen Neuroscience-Aspen's lead product (ANPD001) is currently undergoing investigational new drug (IND)enabling studies for the treatment of sporadic Parkinson disease

- CENTURY THERAPEUTICS- The company's genetically-engineered immune effector cell therapies derived from iPSCs are used to target hematologic and solid cancers.

- T-CiRA- T-CiRA has prepared CAR-T iPS cells (iCART) and plans to start the first-in-human iCART trial

- Cytovia- Cytovia is focusing on cancer immunotherapy by harnessing the power of iPSC CAR NK cells to defeat cancer.

- Heartseed, Inc.- - The company has original technologies throughout the process of manufacturing and delivering iPSC-derived cardiac regenerative medicine

- Megakaryon Corporation- Megakaryon is focused on developing and commercializing the technology to produce and store platelets and red blood cells from iPS cell lines.

- Thyas Co., Ltd.- Thyas develops both "off-the-shelf" allogeneic (others) iPS-T and autologous (yours) iPS$\mathrm{T}$, to bring the ultimate personalized medicines to all patients suffering from solid tumors.

\section{Conclusions}

In this review, ClinicalTrials.gov, the WHO ICTRP, and several country-specific clinical trial databases were consulted to investigate clinical studies involving iPSCs. Our research revealed that numerous present trials entail use of iPSCs but majority merely evaluate the cells, as seen in cases of iPSC-manufacturing or when used for screening purposes; clinical trials in which iPSCs are transplanted into humans in therapeutic capacity, are much less common.

Considering that iPSCs have been known to the world since 2006, and the first iPSC-derived clinical trials were conducted in 2014, it is surprising that such little progress has been made in the development of therapeutic agents using iPSCs, and that iPSCs-derived products have only been administered to humans for approximately 7-8 years. The relative shortage of clinical trials focusing on the administration of iPSC-based therapies could be ascribed to the reported genomic instability of iPSCs [53]. However, a wide range of therapeutics are being developed for various indications, by several therapeutics research and development companies, and it is expected that the development and clinical uptake of iPSC-derived therapeutic alternatives will become increasingly prevalent in the future.

Supplementary Information The online version contains supplementary material available at https://doi.org/10.1007/s12015-021-10262-3.

Authors' Contributions All authors contributed to the study conception and design. Data collection and analysis were performed by Jennifer Yejean Kim, Yoojun Nam. The first draft of the manuscript was written by Jennifer Yejean Kim, Yoojun Nam and Yeri Alice Rim and Jihyeon Ju commented on previous verions of the manuscript. All authors read and approved the final manuscript.

Funding This research was supported by the Basic Science Research Program of the National Research Foundation of Korea (NRF), funded by the Ministry of Education (Grant numbers: NRF-2021R1C1C2004688 and NRF-2019R1A5A2027588, NRF2020R1A2C3004123), and a grant from the Korea Healthcare Technology R\&D Project, Ministry of Health and Welfare, Republic of Korea (Grant numbers: HI16C2177 and HI20C0495).

Availability of Data and Material All data (or sources thereof) relevant to this study are included in the article; further inquiries can be directed to the corresponding author/s.

\section{Declarations}

Ethics Approval Not applicable.

Consent to Participate Not applicable.

Consent for Publication Not applicable.

Conflict of Interest The authors declare no competing interests. Y.N. are employee at YiPSCELL, Inc., and J.H.J. is the employer. J.H.J. is the founder of YiPSCELL. Inc. and also a MD and professor at the Seoul St. Mary's hospital, Catholic University of Korea.

\section{References}

1. Nam, Y., Rim, Y. A., \& Ju, J. H. (2017). Chondrogenic pellet formation from cord blood-derived induced pluripotent stem cells. Journal of Visualized Experiments, (124).

2. Nam, Y., et al. (2017). Cord blood cell-derived iPSCs as a new candidate for chondrogenic differentiation and cartilage regeneration. Stem Cell Research \& Therapy, 8(1), 16.

3. Nam, Y., et al. (2018). Current therapeutic strategies for stem cell-based cartilage regeneration. Stem Cells Int, 2018, 8490489.

4. Yamanaka, S. (2007). Strategies and new developments in the generation of patient-specific pluripotent stem cells. Cell Stem Cell, 1(1), 39-49.

5. Till, J. E., \& McCulloch, E. A. (1961). A direct measurement of the radiation sensitivity of normal mouse bone marrow cells. Radiation research, 14(2), 213-222.

6. Thomson, J. A., et al. (1998). Embryonic stem cell lines derived from human blastocysts. Science, 282(5391), 1145-1147.

7. Takahashi, K., \& Yamanaka, S. (2006). Induction of pluripotent stem cells from mouse embryonic and adult fibroblast cultures by defined factors. Cell, 126(4), 663-676. 
8. Takahashi, K., et al. (2007). Induction of pluripotent stem cells from adult human fibroblasts by defined factors. Cell, 131(5), 861-872.

9. Stadtfeld, M., \& Hochedlinger, K. (2010). Induced pluripotency: History, mechanisms, and applications. Genes \& dev\#elopment, 24(20), 2239-2263.

10. Kimbrel, E. A., \& Lanza, R. (2016). Pluripotent stem cells: The last 10 years. Regenerative Medicine, 11(8), 831-847.

11. Liu, G., et al. (2020). Advances in pluripotent stem cells: history, mechanisms, technologies, and applications. Stem Cell Reviews and Reports, 16(1), 3-32.

12. da Cruz, L., et al. (2018). Phase 1 clinical study of an embryonic stem cell-derived retinal pigment epithelium patch in agerelated macular degeneration. Nature Biotechnology, 36(4), 328-337.

13. Schwartz, S. D., et al. (2015). Human embryonic stem cellderived retinal pigment epithelium in patients with age-related macular degeneration and Stargardt's macular dystrophy: Follow-up of two open-label phase 1/2 studies. Lancet, 385(9967), 509-516.

14. Shroff, G. (2016). Human embryonic stem cell therapy in chronic spinal cord injury: A retrospective study. Clinical and Translational Science, 9(3), 168-175.

15. de Wert, G., \& Mummery, C. (2003). Human embryonic stem cells: Research, ethics and policy. Human Reproduction, 18(4), 672-682.

16. Taylor, C. J., Bolton, E. M., \& Bradley, J. A. (2011). Immunological considerations for embryonic and induced pluripotent stem cell banking. Philosophical Transactions of the Royal Society of London. Series B, Biological Sciences, 366(1575), 2312-2322.

17. Lee, G., et al. (2009). Modelling pathogenesis and treatment of familial dysautonomia using patient-specific iPSCs. Nature, 461(7262), 402-406.

18. Liu, G.-H., et al. (2014). Modelling Fanconi anemia pathogenesis and therapeutics using integration-free patient-derived iPSCs. Nature Communications, 5(1), 1-17.

19. Yoshida, T., et al. (2014). The use of induced pluripotent stem cells to reveal pathogenic gene mutations and explore treatments for retinitis pigmentosa. Molecular brain, 7(1), 1-11.

20. Kondo, T., et al. (2013). Modeling Alzheimer's disease with iPSCs reveals stress phenotypes associated with intracellular A $\beta$ and differential drug responsiveness. Cell Stem Cell, 12(4), 487-496.

21. Duong, T. T., et al. (2018). Use of induced pluripotent stem cell models to probe the pathogenesis of Choroideremia and to develop a potential treatment. Stem Cell Research, 27, 140-150.

22. Kim, C., et al. (2013). Studying arrhythmogenic right ventricular dysplasia with patient-specific iPSCs. Nature, 494(7435), $105-110$.

23. Koci, B., et al. (2017). An impedance-based approach using human iPSC-derived cardiomyocytes significantly improves in vitro prediction of in vivo cardiotox liabilities. Toxicology and Applied Pharmacology, 329, 121-127.

24. Sirenko, O., et al. (2013). Multiparameter in vitro assessment of compound effects on cardiomyocyte physiology using iPSC cells. Journal of Biomolecular Screening, 18(1), 39-53.

25. Hofrichter, M., et al. (2017). Comparative performance analysis of human iPSC-derived and primary neural progenitor cells (NPC) grown as neurospheres in vitro. Stem Cell Research, 25, 72-82.

26. Kondo, T., et al. (2017). iPSC-based compound screening and in vitro trials identify a synergistic anti-amyloid $\beta$ combination for Alzheimer's disease. Cell reports, 21(8), 2304-2312.

27. Ozay, E. I., et al. (2019). Cymerus ${ }^{\mathrm{TM}}$ iPSC-MSCs significantly prolong survival in a pre-clinical, humanized mouse model of Graft-vs-host disease. Stem Cell Research, 35, 101401.
28. Doi, D., et al. (2020). Pre-clinical study of induced pluripotent stem cell-derived dopaminergic progenitor cells for Parkinson's disease. Nature Communications, 11(1), 1-14.

29. Sundberg, M., et al. (2013). Improved cell therapy protocols for Parkinson's disease based on differentiation efficiency and safety of hESC-, hiPSC-, and non-human primate iPSC-derived dopaminergic neurons. Stem Cells, 31(8), 1548-1562.

30. Deinsberger, J., Reisinger, D., \& Weber, B. (2020). Global trends in clinical trials involving pluripotent stem cells: A systematic multi-database analysis. NPJ Regenerative Medicine, 5(1), 1-13.

31. Fung, M., et al. (2017). Responsible translation of stem cell research: An assessment of clinical trial registration and publications. Stem Cell Reports, 8(5), 1190-1201.

32. Monsarrat, P., et al. (2016). An innovative, comprehensive mapping and multiscale analysis of registered trials for stem cell-based regenerative medicine. Stem Cells Translational Medicine, 5(6), $826-835$.

33. Li, M. D., Atkins, H., \& Bubela, T. (2014). The global landscape of stem cell clinical trials. Regenerative Medicine, 9(1), 27-39.

34. Kwon, S. G., et al. (2018). Recent advances in stem cell therapeutics and tissue engineering strategies. Biomaterials Research, 22, 36.

35. Jung, H., et al. (2021). Restoration of osteogenesis by CRISPR/ Cas9 genome editing of the mutated COL1A1 Gene in osteogenesis imperfecta. Journal of Clinical Medicine, 10(14), 3141.

36. Jang, Y., et al. (2019). Development of immunocompatible pluripotent stem cells via CRISPR-based human leukocyte antigen engineering. Experimental \& Molecular Medicine, 51(1), 1-11.

37. Rim, Y. A., et al. (2018). Recent progress of national banking project on homozygous HLA-typed induced pluripotent stem cells in South Korea. Journal of Tissue Engineering and Regenerative Medicine, 12(3), e1531-e1536.

38. Huang, C. Y., et al. (2019). Human iPSC banking: Barriers and opportunities. Journal of Biomedical Science, 26(1), 87.

39. Kanemura, H., et al. (2014). Tumorigenicity studies of induced pluripotent stem cell (iPSC)-derived retinal pigment epithelium (RPE) for the treatment of age-related macular degeneration. PLoS One, 9(1), e85336.

40. Lee, A. S., et al. (2013). Tumorigenicity as a clinical hurdle for pluripotent stem cell therapies. Nature Medicine, 19(8), 998-1004.

41. Bloor, A. J. C., et al. (2020). Production, safety and efficacy of iPSC-derived mesenchymal stromal cells in acute steroid-resistant graft versus host disease: A phase I, multicenter, open-label, doseescalation study. Nature Medicine, 26(11), 1720-1725.

42. Takahashi, M. (2013). Retinal cell therapy using iPS cells. Rinsho Shinkeigaku, 53(11), 1016

43. Takahashi, M. (2016). [Retinal cell therapy using iPS cells]. Nippon Ganka Gakkai Zasshi, 120(3), 210-24; discussion 225.

44. Kawamura, T., et al. (2014). N-glycans: phenotypic homology and structural differences between myocardial cells and induced pluripotent stem cell-derived cardiomyocytes. PLoS One, 9(10), e111064.

45. Mandai, M., et al. (2017). Autologous induced stem-cell-derived retinal cells for macular degeneration. New England Journal of Medicine, 376(11), 1038-1046.

46. Kawamura, M., et al. (2013). Enhanced survival of transplanted human induced pluripotent stem cell-derived cardiomyocytes by the combination of cell sheets with the pedicled omental flap technique in a porcine heart. Circulation, 128(11 Suppl 1), S87-94.

47. Kawamura, T., et al. (2015). Structural changes in N-Glycans on induced pluripotent stem cells differentiating toward cardiomyocytes. Stem Cells Translational Medicine, 4(11), 1258-1264.

48. Domae, K., et al. (2021). Clinical outcomes of autologous stem cell-patch implantation for patients with heart failure with 
nonischemic dilated cardiomyopathy. Journal of the American Heart Association, 10(13), e008649.

49. Takahashi, J. (2020). iPS cell-based therapy for Parkinson's disease: A Kyoto trial. Regenerative Therapy, 13, 18-22.

50. Hermanson, D. L., et al. (2016). Induced pluripotent stem cellderived natural killer cells for treatment of ovarian cancer. Stem Cells, 34(1), 93-101.

51. Zhu, H., et al. (2020). Pluripotent stem cell-derived NK cells with high-affinity noncleavable CD16a mediate improved antitumor activity. Blood, 135(6), 399-410.
52. Cichocki, F., et al. (2020) iPSC-derived NK cells maintain high cytotoxicity and enhance in vivo tumor control in concert with T cells and anti-PD-1 therapy. Science Translation Medicine, 12(568).

53. Ji, P., et al. (2016). Induced pluripotent stem cells: Generation strategy and epigenetic mystery behind reprogramming. Stem Cells International, 2016, 8415010.

Publisher's Note Springer Nature remains neutral with regard to jurisdictional claims in published maps and institutional affiliations. 